\title{
Recent advances in nanostructured biomimetic dry adhesives
}

\section{Andras Pattantyus-Abraham, Jeffrey Krahn and Carlo Menon*}

MENRVA Research Group, School of Engineering Science, Simon Fraser University, Burnaby, BC, Canada

Edited by:

Cecilia Laschi, Scuola Superiore

Sant'Anna, Italy

Reviewed by:

Lorenzo Masia, Nanyang

Technological University, Singapore

Renato Vidoni, Free University of

Bozen-Bolzano, Italy

loannis Andrea leropoulos, University

of the West of England, UK

*Correspondence:

Carlo Menon, MENRVA Research

Group, School of Engineering

Science, Simon Fraser University,

8888 University Drive, Burnaby, BC

V5A 1S6, Canada

e-mail:carlo_menon@sfu.ca

\begin{abstract}
The relatively large size of the gecko and its ability to climb a multitude of structures with ease has often been cited as the inspiration upon which the field of dry adhesives is based. Since 2010, there have been many advances in the field of dry adhesives with much of the new research focusing on developing nanoscale and hierarchical features in a concentrated effort to develop synthetic gecko-like dry adhesives which are strong, durable, and self-cleaning. A brief overview of the geckos and the hairs which it uses to adhere to many different surfaces is provided before delving into the current methods and materials used to fabricate synthetic gecko hairs. A summary of the recently published literature on bio-inspired, nanostructured dry adhesives is presented with an emphasis being placed on fabrication techniques.
\end{abstract}

Keywords: biomimetic, dry adhesive, nanostructure, mold, gecko adhesive

\section{INTRODUCTION}

The field of biomimetic dry adhesives has flourished in recent years. From the earliest report on synthetic, gecko-inspired, adhesives (Geim et al., 2003; Sitti and Fearing, 2003), there has been substantial progress in understanding the theory, materials, and fabrication processes underlying dry adhesion. The optimization of adhesive properties involves the synergistic exploration of solid mechanics, materials synthesis and properties, interface properties, micro- and nanostructuring, all supported by the high level of function observed in biological examples.

Interest in biomimetic dry adhesives has been driven, in many cases, by the desire to achieve climbing robots and has been inspired largely by observation of the Tokay gecko which may weigh up to $300 \mathrm{~g}$ and reach lengths up to $35 \mathrm{~cm}$ - approximately the same size and length as many climbing robots using synthetic dry adhesives as an attachment method (Daltorio et al., 2006; Kim et al., 2008; Krahn et al., 2011). The adhesion strength produced by the gecko foot pad can reach $10 \mathrm{~N}$ for a $100 \mathrm{~mm}^{2}$ (Autumn et al., 2000) area and is used on a wide variety of surfaces providing a yet unachieved goal for synthetic dry adhesion strength and utility.

The gecko foot adhesion has been shown to rely on van der Waals forces (Autumn et al., 2002). In order to maximize the interfacial contact area, the gecko foot depends on the multiscale structure of the hierarchical keratin foot hairs, which are located on the toe pad of the gecko and are reported to have non-matting and self-cleaning properties (Hansen and Autumn, 2005). Each hierarchical layer allows the gecko foot to adapt and attach to rough and bumpy surfaces by compressing or conforming to varying levels of surface roughness. As is shown in Figure 1, the base layer of the hierarchical structures is composed of soft ridges on the surface of the geckos toes called lamellae which are approximately
$1-2 \mathrm{~mm}$ in length and are easily compressed for improved contact on rough surfaces. From the surface of the lamellae extend curved hairs or setae which range from 5 to $10 \mu \mathrm{m}$ wide and from 30 to $130 \mu \mathrm{m}$ long. The ends of the setae are covered with spatula whose tips are approximately $500 \mathrm{~nm}$ long, $10 \mathrm{~nm}$ thick, and $200-300 \mathrm{~nm}$ wide and are able to conform to small-scale surface roughness. (Bhushan and Sayer, 2007) (Figure 2). The mechanics of attachment and detachment also play a significant role in determining the adhesive performance of the gecko (Autumn et al., 2006b).

Top-down microfabrication techniques have been successfully applied to the creation of highly functional dry adhesives for smooth surfaces (Gorb et al., 2007; Sameoto and Menon, 2010; Song et al., 2013). The present review however provides a summary of the recent progress in the area of nanostructured dry adhesives since 2010. The further challenge of incorporating hierarchical structures involving nanometer-scale components is also given attention. We have excluded carbon nanotube based adhesives, which are in fact very important, but are covered by a recent review (Hu et al., 2013). Similarly we have excluded adhesion models which are discussed in detail in another recent review (Zhou et al., 2013). In particular, we focus on recent reports on fabrication processes.

\section{OVERVIEW OF CRITERIA FOR BIOMIMETIC DRY ADHESIVES}

In order to mimic the gecko, a dry adhesive should meet the following criteria (Autumn, 2007):

1. adhesion through van der Waals interactions

2. anisotropic adhesion

3. a high pull-off to preload ratio

4. low detachment force when required

5. self-cleaning 


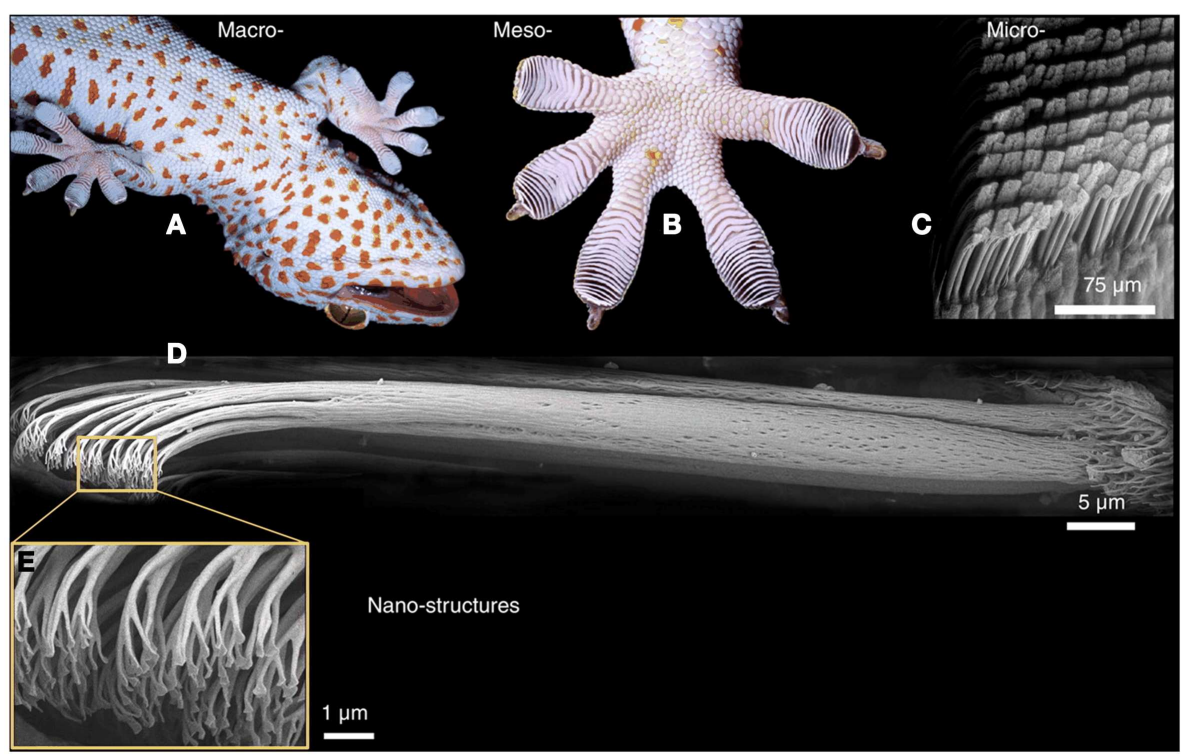

FIGURE 1 | Structural hierarchy of the gecko adhesive system. (A,B) Gecko toe pads. (C) Gecko setae. (D) A single seta of the tokay gecko. (E) Gecko spatulae. From Autumn et al. (2006a).
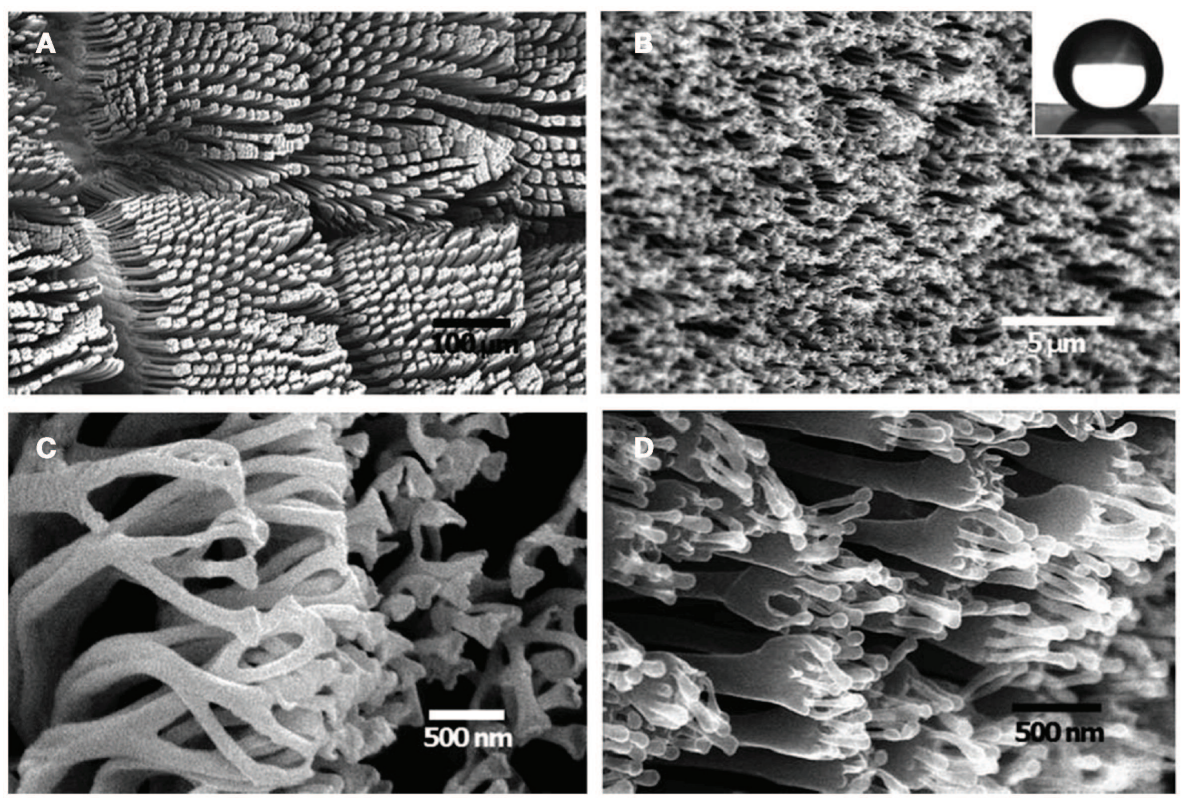

FIGURE 2 | SEM images of a Tokay Gecko (Gekko gecko) (A,C) and fabricated hierarchical polystyrene (PS) nanohairs with high aspect ratio (AR) (B,D) Inset in (B) water contact angle of the elongated hierarchical PS nanohairs. From Lee et al. (2012).

6. anti-self-matting/self-adhesion

7. a low to no adhesion state in the absence of shear

Wet pressure-sensitive adhesives conventionally have a Young's modulus below $100 \mathrm{kPa}$, whereas geckos achieve similar effective modulus from beta-keratin (Autumn et al., 2006a), which has a much higher bulk modulus in the gigapascal range. The foot hair geometry is essential to achieve this dramatic difference in modulus, and enables the maximization of contact area. As described by Jeong and Suh (2009), four key structural features are considered to be advantageous for modulus translation:

1. High aspect ratio (AR) features

2. Angled/tilted features

3. Multiple length scale hierarchical features

4. Spatula or mushroom cap-terminated features 
For practical adhesive applications, additional criteria of importance are: area fill fraction, cost of mold preparation, mold durability, and scalability to large-area production. Also, the actual ability to maintain high adhesion in the presence of contamination, or the ability to easily shed contamination, is crucial for applications such as climbing robots.

\section{NANOSTRUCTURE FABRICATION PROCESSES}

Outside of CNT-based approaches, which involve directed growth of nanotubes, most practical nanostructured dry adhesive fabrication processes are based on a nanocasting process to form nanowires/nanofibers. The overall process encompasses mold preparation, mold pre-treatment, material infusion, demolding, and post-molding treatments of the demolded adhesive. Each step has important variables that may need to be optimized for a given molding strategy and material system.

A general representation of the nanocasting process is shown in Figure 3. The initial form may either be a positive or negative replica of the desired final product. In the case of a positive replica,

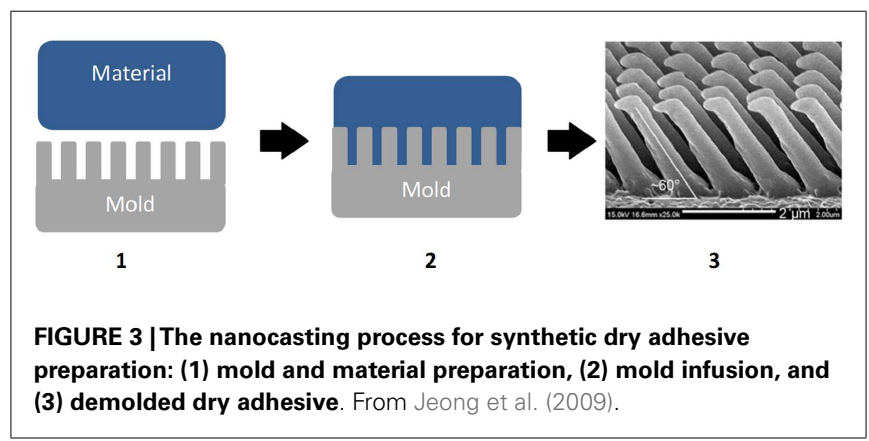

an additional negative replication step is then necessary, but this can be advantageous for minimizing damage to the master mold.

An important exception in the recent literature is the application of two-photon lithography to directly write adhesive microand nanostructures (Röhrig et al., 2012). This approach allows unprecedented control over the structural configuration of an adhesive (Figure 4), allowing high aspect ratios, tilted, and hierarchical structures, as well as mushroom caps. Presumably, the resulting structures could be transferred into other widely used polymers via a molding process. However, one limitation of twophoton lithography is that the process is sequential and large-area exposures may be very time-consuming.

\section{Nanomold preparation}

The mold selection or fabrication step is often the most important one. The key structural components of the fibers (AR, tilt, hierarchy, cap) are determined at this stage. Furthermore, the ability to scale up to macroscopic dimensions is important for all but purely fundamental studies, and is largely determined by the mold.

On one hand, many commercially available nanoporous membranes may be used as inexpensive molds, at the expense of limiting the parameter space to the offering of the membrane producer. On the other hand, cleanroom lithographic processes may be expensive and scale poorly.

Several different molding techniques have been used to create nanostructures including melting, UV-crosslinking, polymerization, capillary force nanoimprinting, and twophotopolymerization.

The melting technique involves applying sufficient heat to melt cyclic olefin copolymer (COC), Teflon AF, polypropylene (PP), or low density polyethylene (LDPE) or high density polyethylene (HDPE). In some cases a hydraulic press or injection molding is

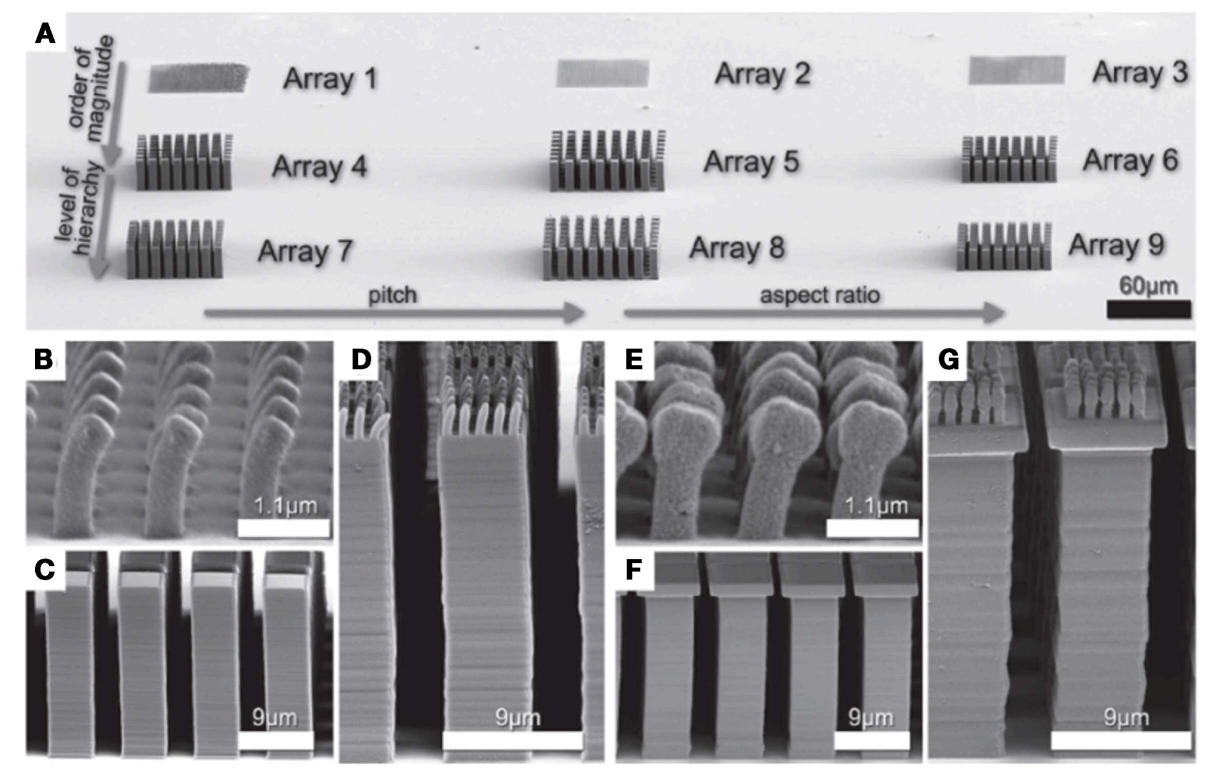

FIGURE 4 | (A) Several gecko-inspired arrays fabricated by 3D direct laser writing. (B) SEM image of array 1, which contains small single level structures with a width of $500 \mathrm{~nm}$. (C) The SEM image shows array 4, which contains pillars of $5 \mu \mathrm{m}$ width. (D) Array 7 consists of the array 1 on top of array 4 . (E-G) Show corresponding arrays with mushroom-shaped tips. The scale bars refer to the periodicity of the arrays (Röhrig et al., 2012). 
used to force the melted polymer into the mold cavities formed by membranes, porous anodic aluminum oxide (AAO), silicon nanowires, or molds fabricated by electron beam lithography (EBL) or other technique. A major limitation of using the melting technique is the fabrication or procurement of the molding cavity as commercial injection molding equipment and hydraulic presses are readily available.

Another approach to creating nanostructure arrays is to cast a UV-curable polymer such as soft or hard polyurethane acrylate (PUA) into the mold cavity. UV-curable polymers typically require only a few seconds of exposure to UV light in order to achieve a rapid transition from resin to polymer. Nanostructures have also been fabricated using capillary force nanoimprinting using AAO as a template.

A summary of the nanostructuring techniques is presented in Table 1 .

\section{MATERIALS}

Whereas microstructured dry adhesives rely on softer materials such as PDMS, with a typical bulk modulus below $5 \mathrm{MPa}$, nanostructured dry adhesive rely on stiffer materials. A wide variety of polymers have been reported, and there is a natural bias toward easily available and readily processable formulations. The polymers used in the recent literature are summarized in Table 2.

\section{MOLDING TECHNIQUES}

Porous AAO has been used extensively to prepare nanostructured materials since the preparation of ordered pore arrays was reported (Masuda and Fukuda, 1995). It is commercially available as a filtration membrane with a wide range of pore sizes and thicknesses (Whatman Inc.). The laboratory preparation process is also relatively simple, requiring only high purity aluminum foil, a simple electrolyte solution such as dilute sulfuric or oxalic acid, and a DC voltage supply. For membrane fabrication, pore spacing is controlled via the applied potential with self-ordered pore lattices only being obtained with specific voltages and electrolytes. Ordered

Table 2 | Polymers used for nanostructured dry adhesives.

\begin{tabular}{|c|c|c|}
\hline Polymer & $\begin{array}{l}\text { Modulus } \\
\text { (GPa) }\end{array}$ & Reference \\
\hline IP-G 780 & 4 & Röhrig et al. (2012) \\
\hline $\begin{array}{l}\text { Polyurethane acrylate (PUA), } \\
\text { hard }\end{array}$ & $0.32-1.3$ & $\begin{array}{l}\text { Jeong et al. (2010a), Jeong } \\
\text { and Suh (2012) }\end{array}$ \\
\hline Polyurethane acrylate, soft & 0.02 & Jeong and Suh (2012) \\
\hline Polyethylene (PE) & $0.4-1.0$ & Palacio et al. (2013) \\
\hline Low density polyethylene (LDPE) & 0.2 & Lee et al. (2011) \\
\hline $\begin{array}{l}\text { High density polyethylene } \\
\text { (HDPE) }\end{array}$ & 0.4 & $\begin{array}{l}\text { Gillies and Fearing (2011), } \\
\text { Lee and Fearing (2012) }\end{array}$ \\
\hline Polypropylene (PP) & $1.3-2$ & $\begin{array}{l}\text { Gillies and Fearing (2011), } \\
\text { Rodríguez et al. (2013), Lee } \\
\text { and Bushan (2012) }\end{array}$ \\
\hline Teflon AF & 1.5 & Izadi et al. (2013) \\
\hline Polyimide (PI) & - & Liu et al. (2012) \\
\hline Polycarbonate (PC) & 2.2 & Ho et al. (2011) \\
\hline Polystyrene (PS) & 3.2 & Lee et al. (2012) \\
\hline Poly-DL-lactide (PDLLA) & $0.3-2.3$ & Rodríguez et al. (2013) \\
\hline $\begin{array}{l}\text { Cyclic olefin copolymer TOPAS } \\
8007 \text { (COC) }\end{array}$ & 2.6 & $\begin{array}{l}\text { Matschuk and Larsen } \\
\text { (2013) }\end{array}$ \\
\hline
\end{tabular}

Table 1 | Summary of nanostructured dry adhesives in recent literature.

\begin{tabular}{|c|c|c|c|c|c|c|}
\hline Structuring technique & Reference & Polymer & Preparation & Hierarchical? & Diameter (nm) & Aspect ratio \\
\hline Si mold, optical lithography & Jeong et al. (2010a) & h-PUA & UV-crosslink & No & 400 & 5 \\
\hline Si mold, optical lithography & Jeong and Suh (2012) & s-PUA; h-PUA & UV-crosslink & No & 700 & 3.6 \\
\hline \multirow[t]{2}{*}{ 3D direct laser writing } & Röhrig et al. (2012) & IP-G 780 & Two-photon & No & 500,5600 & $3-4$ \\
\hline & & & polymerization & Yes & $5600 / 500$ & $3-4 / 3-5$ \\
\hline Track etch PC mold & Palacio et al. (2013) & $\mathrm{PE}$ & Melt & Yes & $5000 / 600$ & 6,50 \\
\hline Track etch PC mold & Gillies and Fearing (2011) & HDPE; PP & Melt & No & 600 & 33 \\
\hline Track etch PC mold & Lee et al. (2011) & HDPE & Melt & No & 300 & 12,60 \\
\hline Track etch PC mold & Lee and Bushan (2012) & $\mathrm{PP}$ & Melt & No & $50,100,600,5000$ & $6,50,300,600$ \\
\hline Track etch PC mold & Rodríguez et al. (2013) & PP; PDLLA & Melt & No & 200 & 10 \\
\hline Microsphere lithography on Si & Lee et al. (2011) & LDPE & Melt & No & $250-900$ & $1-10$ \\
\hline AAO mold & Izadi et al. $(2012,2013)$ & Teflon AF & Melt & Yes & 200 & 80 \\
\hline AAO mold & Liu et al. (2012) & $\mathrm{Pl}$ & Polymerization & No & $100-200$ & $30-60$ \\
\hline \multirow[t]{3}{*}{ AAO mold } & Ho et al. (2011) & PC & Capillary force & Yes & $280 / 90$ & $10 / 9$ \\
\hline & & & nanoimprinting & & $280 / 110$ & $10 / 6$ \\
\hline & & & & No & 280 & 23 \\
\hline \multirow[t]{2}{*}{ AAO mold } & Lee et al. (2012) & PS & Melt & Yes & $380 / 100$ & $2.4,1.8$ \\
\hline & & & & & $3000 / 70$ & 10,7 \\
\hline Ni mold, EBL & Matschuk and Larsen (2013) & $\mathrm{COC}$ & Melt & No & 40 & $1-2$ \\
\hline
\end{tabular}


arrays have been demonstrated with 50-500 nm pitch (Nielsch et al., 2002), with pore diameters typically $50-70 \%$ of this value.

The pore arrays are also close-packed in micron-scale domains and this leads to a near ideal filling fraction, which is important both for maximizing adhesive contact area as well as preventing fiber collapse.

Since the pore depth is determined by anodization time, very high and precisely controlled aspect ratio molds can be prepared $(\mathrm{AR}>1000)$. The resulting pore diameter may be tuned over a modest range via etching in dilute acids.

Furthermore, it has been shown that the anodization voltage may be altered with time to create hierarchical structures (Ho et al., 2011; Izadi et al., 2012; Lee et al., 2012) (Figure 5) and a membrane stacking approach can also be used to obtain a mold with multiple length scales. An extra level of hierarchy has also been introduced through the fingering instability during polymer infusion (Izadi et al., 2013).

As AAO may be etched away readily with dilute acids, a destructive demolding process can be readily effected and, since the mold production and material cost is relatively low, this could be viable for many applications.
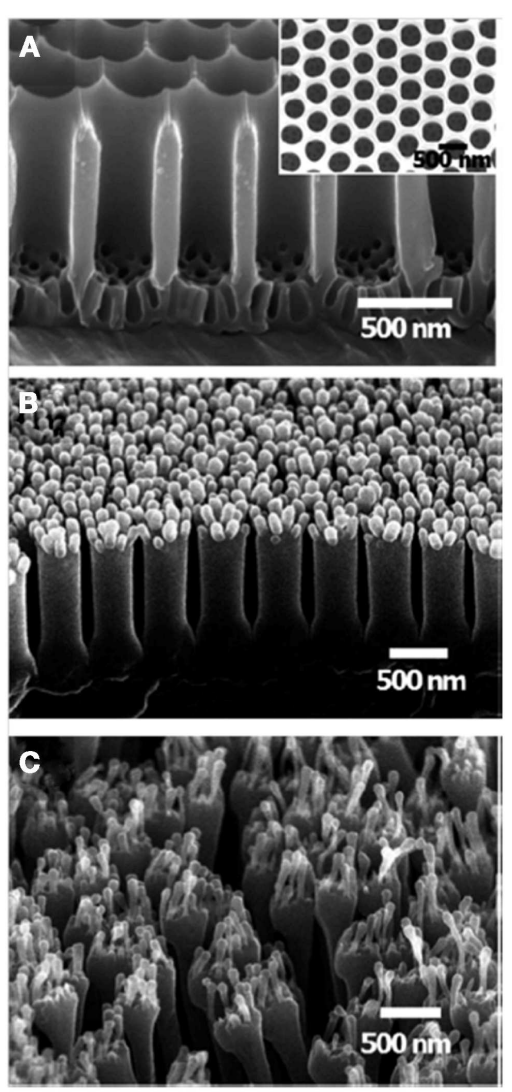

FIGURE 5 | SEM images of (A) the multi-branched AAO template (inset, top view), (B) the PS nanohairs replicated by removing the template during the wet etching process (denoted DLW), and (C) elongated hierarchical PS nanohairs replicated using the surface-modified template and peel-off process (denoted DL) (tilt angle $\mathbf{4 5}^{\circ}$ ). From Lee et al. (2012).
Anodic aluminum oxide molds however do not readily lend themselves to tilted structures, but there is an initial report of slanted fibers obtained by changing the angle of release of the template (Lee et al., 2012). Also, precise control of the mold tip shape has not yet been demonstrated.

Even though commercial hydraulic presses and injection molders are readily available, AAO membranes are typically smaller than $50 \mathrm{~mm}$ in diameter which places a limitation on the ability to scale up production to large scale sheets to enable nanostructures to become a commercially viable product.

Polycarbonate etch-track membranes are also available commercially as filtration membranes, which has enabled their continued widespread use as a mold for dry adhesives (Gillies and Fearing, 2011; Lee and Bushan, 2012; Lee and Fearing, 2012; Palacio et al., 2013; Rodríguez et al., 2013). The track-etch process, based on selectively dissolving parts of a polymer membrane exposed to nuclear radiation, allows for a very wide range of pore diameters ranging from $50 \mathrm{~nm}$ to $12 \mu \mathrm{m}$ (Millipore, MA, USA), with aspect ratios up to 1000 .

Unfortunately, ion tracks are not ordered and the fill fraction of pores is relatively low $(<20 \%)$ and higher fill fractions would lead to a broader distribution of pore sizes due to track overlaps. In principle, tilted structures are possible with track-etch membranes, but access to a suitable source of nuclear radiation would be a limiting factor.

Due to the large range of diameters, hierarchical micro/ nanomold stacks are possible and have been demonstrated (Lee and Bushan, 2012) (Figure 6). Even though the macroscopic utility of these structures is hampered by the low fill fraction, they still enable local testing of adhesive properties via scanning probe techniques.

Polycarbonate etch-track membranes may currently offer the best choice for large scale production of adhesive nanostructures as a wide range of sheet sizes are available commercially and could relatively easily be paired with hot embossing equipment.

\section{Optical lithography}

Optical lithography is a conventional microfabrication technique which continues to be exploited in combination with reactive ion etching (RIE) to achieve submicron features. While the technique is limited to the size of the Si wafer, the use of different masks in principle allows the precise control of pitch, diameter, and cross-section.

With the innovation of tilted RIE (Jeong et al., 2009), angled structures are also achievable (Figure 7). Finally, the shape of the tip may be controlled via the RIE process (Jeong et al., 2009) or via post-molding modifications (Jeong and Suh, 2012).

\section{Electron beam}

Electron beam lithography allows precise control of mold geometry down to the sub-100 $\mathrm{nm}$ regime, and this capability may yet allow it to deliver important insights since large areas can be patterned in modern tools. Relatively high aspect ratios are possible in principle because the resist thickness can reach $2000 \mathrm{~nm}$. However, high beam energy is required to maintain small feature diameter and in practice this would reduce the aspect ratio to below 100 . 


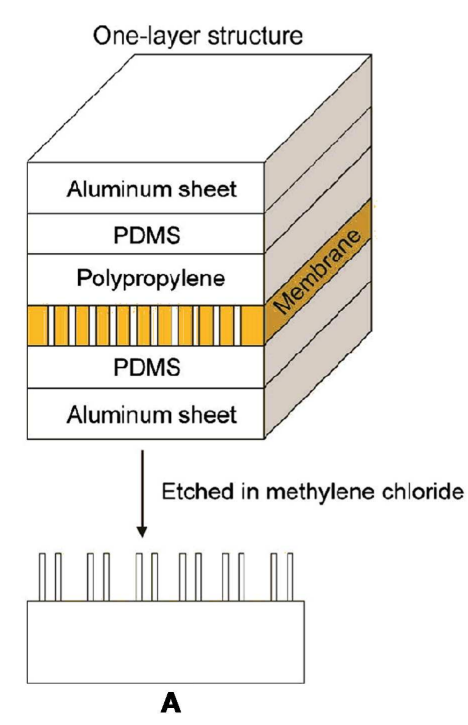

FIGURE 6 | Sample fabrication processes for (A) one-layer structure and (B) two-layer (hierarchical) structure using one and two membranes in the stack, respectively. After heating the stacks in an oven, the membranes
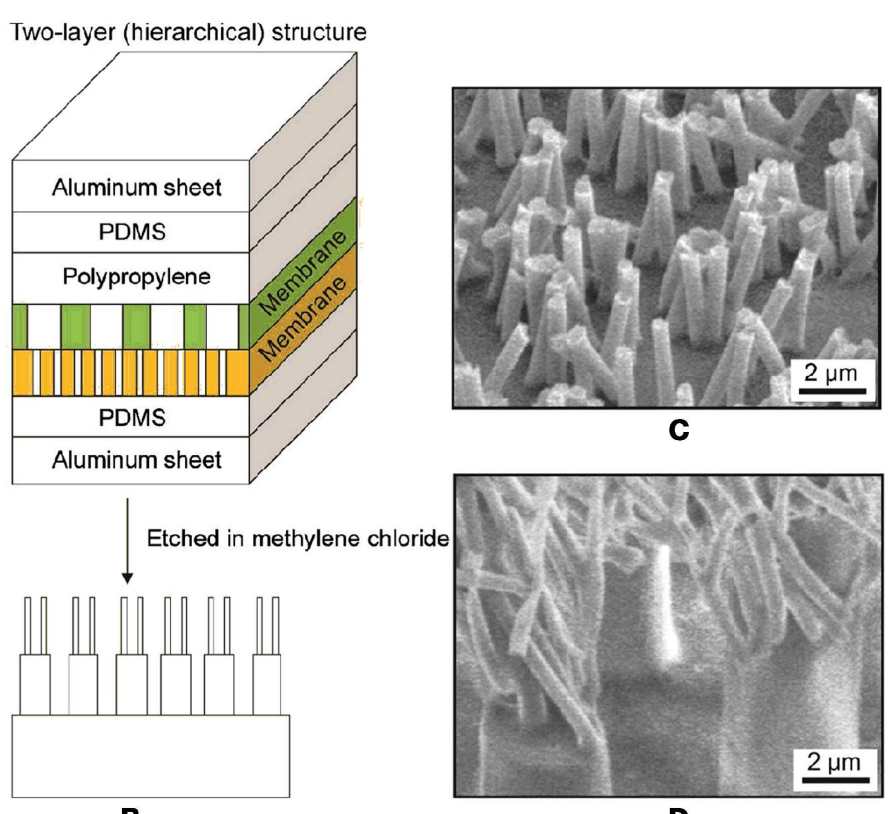

D
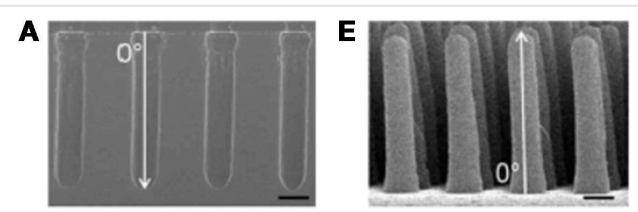

B
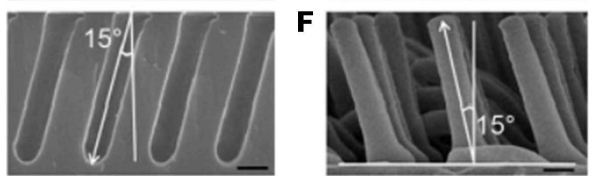

C

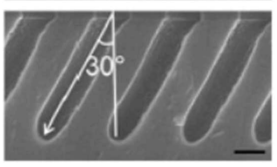

D

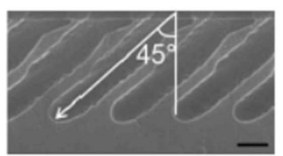

FIGURE 7 | (A-D) SEM images of the Si master substrates, prepared via optical lithography and RIE, having angled etch profiles of $0^{\circ}, 15^{\circ}, 30^{\circ}$, and $45^{\circ}$, respectively. (E-H) SEM images of the h-PUA nanohairs, $2 \mu \mathrm{m}$ length and $400 \mathrm{~nm}$ diameter, replicated from the masters shown in (A-D). Scale bar $=400 \mathrm{~nm}$. From Jeong et al. (2010a).

Electron beam lithography has also been successfully applied to prepare dry adhesives directly from EBL resists (Tsai et al., 2011) and, furthermore, a nanomold was prepared by overcoating the EBL pattern with Ni (Matschuk and Larsen, 2013). Tilted and

are etched using methylene chloride to obtain the nanostructured samples. (C) SEM image of $600 \mathrm{~nm}$ one-layer structure, and (D) two-layer structure made from polypropylene. Lee and Bushan (2012). capped structures have also been demonstrated in metal structures (Zhang et al., 2012) without conversion to an actual dry adhesive (Figure 8).

\section{Microsphere lithography}

Microsphere lithography is based on the use of close-packed, micron to submicron colloidal particles that can be transferred to the surface of a target substrate. The pitch of the pattern is determined by the particle diameter where the pattern openings are tuned by RIE of the colloidal particles. Areas as large as $100 \mathrm{~mm}$ Si wafers may be coated with a uniform layer.

A very novel integration of microsphere lithography was presented recently (Lee et al., 2011), where it was combined with metal-catalyzed electroless etching of $\mathrm{Si}$ to achieve high AR Si posts, which were then used to create a negative mold for nanocasting (Figure 9).

The fill fraction of the final features is high due to the closely packed nature of the microsphere layer. However, the fill fraction diminishes as the microspheres are etched to tune their diameters. In principle, this could be circumvented through the use of different microsphere diameters.

The aspect ratio achievable through microsphere lithography is entirely dependent on the pattern transfer process and, at present, the highest demonstrated aspect ratio is 10 , but it may be possible to extend this further.

Hierarchical structures may be possible with microsphere lithography, if the nanomold is combined with a separately produced micromold based on larger colloidal particles.

In the published implementation, both the Si positive mold and the negative replica are single-use since they are removed by 

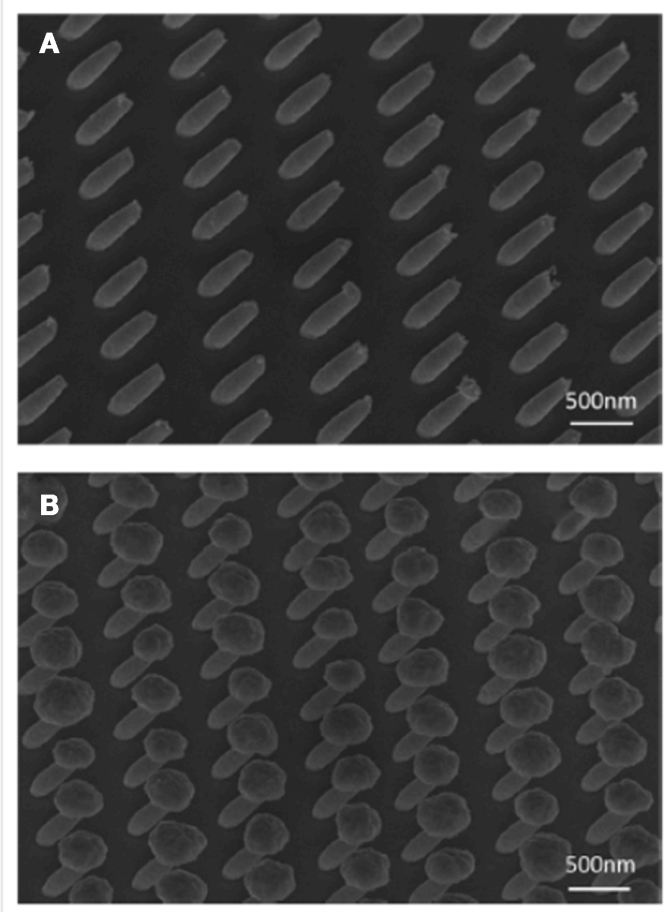

FIGURE 8 | SEM images of $45^{\circ}$ tilted Au pillar arrays by electroplating into tilted hole arrays in electron beam resist. (A) Tilted pillars of $300 \mathrm{~nm}$ diameter and $750 \mathrm{~nm}$ length without Au over-plating. (B) Mushroom-shaped Au pillar array due to over-plating. The images were taken at $45^{\circ}$ viewing angle. From Zhang et al. (2012).

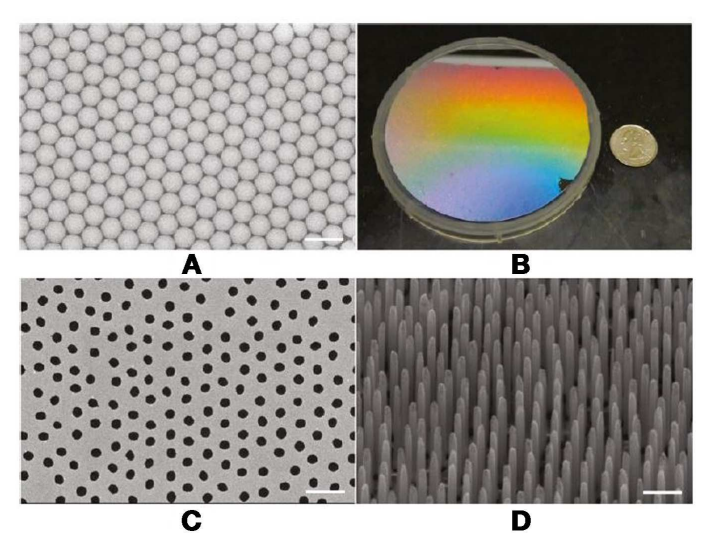

FIGURE 9 | SEM image of PS microsphere array (A) showing well-ordered structure in microscale. Optical image of a 4 " wafer (B), showing uniform PS array. Au-coated patterned Si substrate defined by areas not previously covered by the plasma-etched microspheres (C). Slight disordering is induced during the plasma etching process. Au-coated region becomes a catalytic site during metal-assisted electroless etching, which results in well-defined vertical SiNW structures ( $45^{\circ}$ tilt view) (D). Scale bar $=2 \mu \mathrm{m}$ in all SEM images. From Lee et al. (2011).

chemical etching. It would be more efficient to avoid these destructive demolding steps, possibly through the use of a mold release layer.

\section{MOLD TREATMENTS}

\section{Surface treatments}

The use of mold release agents is common with macroscopic molds, and can be even more critical on the nanoscale, due to the large surface area to volume ratio of the molded features (Matschuk and Larsen, 2013) where the interfacial forces can cause demolding failure and possibly limited reusability of the mold.

For processes using mold surface treatments, the as-fabricated mold often needs a further treatment to ensure proper demolding of the chosen dry adhesive material. The most common strategy is to employ a low-energy release layer, such as a fluoroalkylsilane. This can dramatically improve the nanostructure yield (Matschuk and Larsen, 2013).

\section{Mold infusion}

The introduction of the target material into a nanomold can be a simple or intricate process depending on the infusing material and the aspect ratio of the mold features. When pre-synthesized polymers are used, the infusion process is driven by a combination of pressure and temperature. Heating above the glass transition temperature of the polymer is required to allow for flow to occur and this process can be relatively time-consuming (up to several hours), which is less attractive for rapid production of large volumes.

In situ polymerization and crosslinking is necessary for many materials, such as rubbers (polyurethane, polyimide), which do not melt and cannot be dispersed in a solvent. Also, diffusion of the precursor monomers or oligomers into the mold cavity may be substantially quicker than for pre-synthesized polymers. Conversely, the material properties will depend on polymerization conditions confined on the nanoscale, and may deviate substantially from bulk properties.

Capillary force lithography has been employed to great advantage by Suh and co-workers (Kwak et al., 2010; Jeong and Suh, 2012). This approach doesn't require high pressures but instead relies on capillary forces in the nanoscale features of the mold to induce infiltration. More recently, capillary force assisted nanoimprinting has been reported (Ho et al., 2011).

\section{Demolding}

The demolding step is a critical one for nanocasting, due to the high area of interaction between the mold and the replica. The mold may be removed destructively via a solvent (Gillies and Fearing, 2011; Lee and Bushan, 2012; Lee and Fearing, 2012; Palacio et al., 2013; Rodríguez et al., 2013), wet chemical etching (Lee et al., 2011; Izadi et al., 2012; Liu et al., 2012), or by peeling the replica away (Ho et al., 2011; Lee et al., 2012). While the wet etching approach imparts minimal distortion to the replica, it is known that the drying step that follows wet processing leads to clumping of nanofibers (Kustandi et al., 2007).

The peeling approach is of interest since mold fabrication may be expensive and/or time-consuming. Peeling has been used successfully for Si molds (Jeong and Suh, 2012; Jeong et al., 2010a) and is certainly the case for EBL-generated molds, and a detailed study on mold performance and reliability has been carried out (Matschuk and Larsen, 2013). 

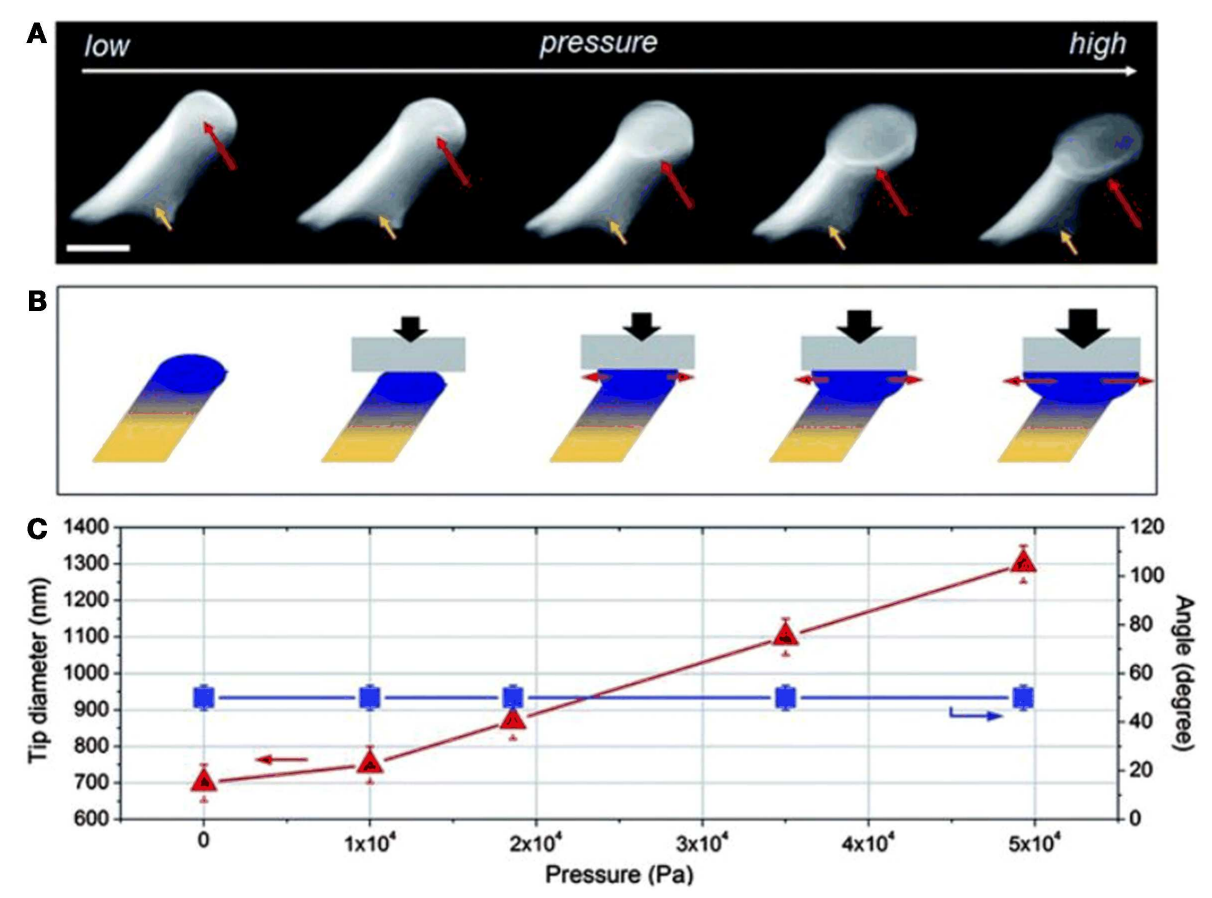

FIGURE 10 | (A) Tilted SEM images of angled, PUA nanopillars with different tip shapes from round to increasingly enlarged mushroomlike tips. The tip diameters of nanopillars increased with the increase of applied pressure. Tip diameters of nanopillars, from left to right, are $700,750,870,1100$, and $1300 \mathrm{~nm}$, respectively. Length of the nanopillar is $2.5 \mathrm{~mm}$ and leaning angle is $50^{\circ}$. (B) A schematic illustration showing the tip shape transformation of a nanopillar with gradually increased mechanical loads. (C) Plots of tip diameter and leaning angle changes as a function of applied pressure. From Jeong and Suh (2012).

\section{POST-MOLDING TREATMENTS}

Both structure and surface chemistry of a nanostructured dry adhesive may be modified after demolding. In the first case, some desired shapes such as mushroom caps may be difficult or impossible to demold. A very good example of post-molding treatments involved partially curing PUA (Jeong and Suh, 2012) within a Si mold, then applying pressure to flatten the fiber tips (Figure 10).

In one case, RIE has been used to remove a thin capping layer on molded fibers, used to prevent clumping during solvent removal of the mold (Rodríguez et al., 2013).

One area that is still ripe for further exploration for nanostructured dry adhesives are modifications of the surface chemistry after micro/nanostructuring is complete, such as the use of fluoroalkylsilane to modify the PI surface (Liu et al., 2012). Fluoroalkylsilane or other treatments may allow modification of the surface energy for specific applications, e.g., for enhanced adhesion, resistance to certain chemicals, or self-cleaning properties.

\section{MEASUREMENTS ON NANOSCALE DRY ADHESIVES}

Local nanoscale testing methods are beginning to yield some insight into the adhesion strength of nanoscale structures but it is difficult to compare independent results without a standardized testing method and standardized reporting. Currently, local nanoscale testing is most often being performed with an atomic force microscope (AFM) (Jeong and Suh, 2012; Lee and Bushan, 2012; Lee et al., 2012; Palacio et al., 2013). Macroscopic testing on the other hand has been performed using linear stages and load cells (Gillies and Fearing, 2011; Izadi et al., 2013), universal testing machines (Ho et al., 2011; Rodríguez et al., 2013), a triboindenter (Northern and Turner, 2005), a hanging test (Jeong et al., 2009; Jeong et al., 2010a,b), or pulley system (Lee et al., 2011) with the method used depending on equipment availability and whether normal adhesion, shear adhesion, or a combination of the two is being reported. Macroscopic testing requires careful quantification of actual contact area, especially due to the high stiffness of the bulk polymer supporting the nanostructures.

The effect of tilt angle on adhesion was further confirmed (Jeong et al., 2010b) and it has been shown nanoscale tip shape modifications also enhances adhesion (Jeong and Suh, 2012; Röhrig et al., 2012).

Similarly, the effect of aspect ratio: too high and fibers will collapse, too low and adhesion is also poor and also applies to nanoscale features (Lee et al., 2011; Röhrig et al., 2012).

Adhesive forces in the nanoscale can be very high and material limits may be exceeded (Gillies and Fearing, 2011) indicating that better materials may be needed for improved wear resistance for long term usage.

In at least one material, very high adhesion in nanostructured hierarchical Teflon AF samples may indicate a mechanism other than van der Waals forces, such as contact electrification (Izadi et al., 2012, 2013). Further investigation is needed in order to take advantage of these and possibly other surface effects. 
Table 3 | Summary of adhesion results.

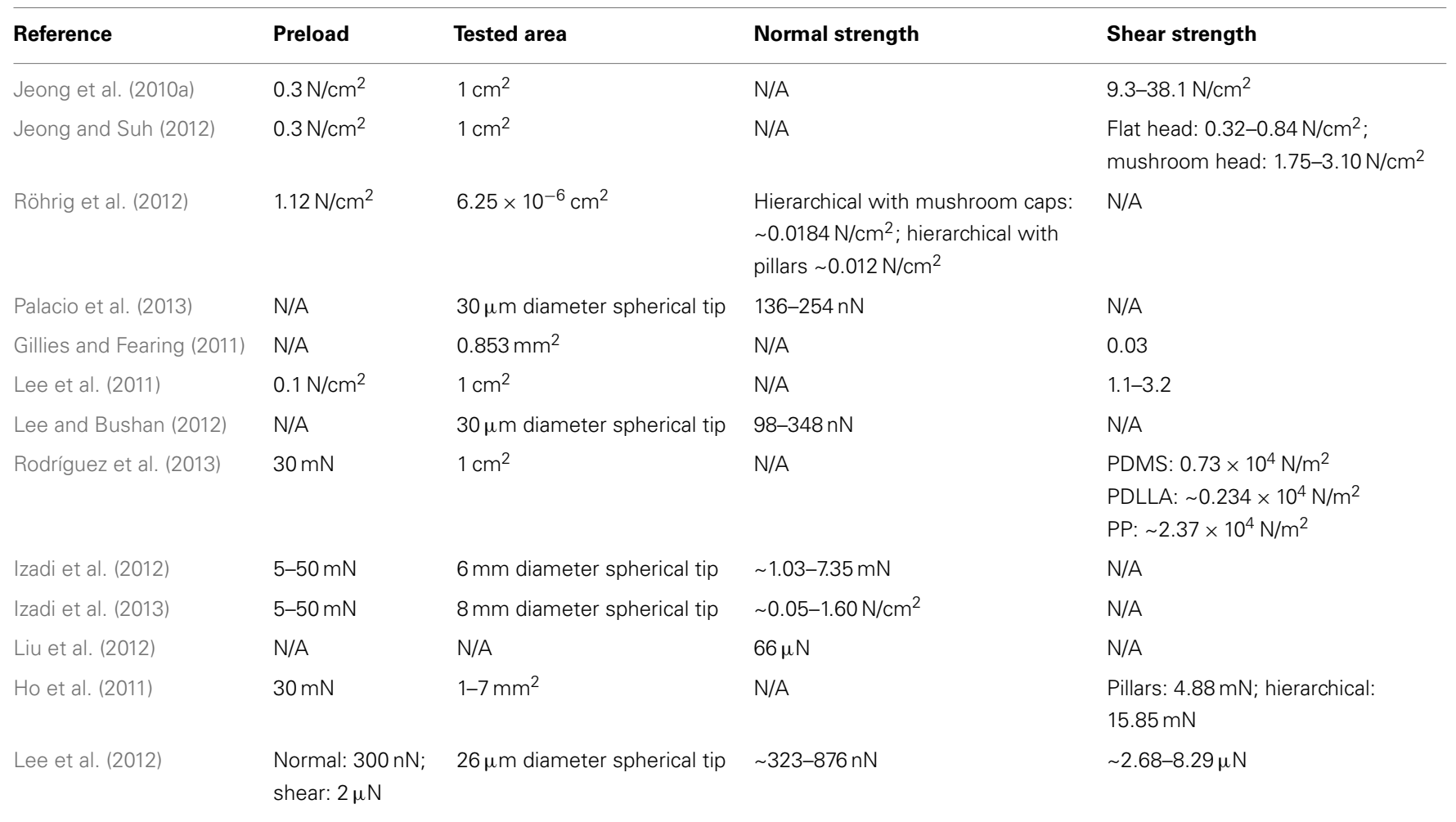

Self-cleaning effects have been tested (Lee and Bushan, 2012; Lee and Fearing, 2012), however general self-cleaning currently remains elusive.

A summary of recent adhesion results are shown in Table 3.

\section{SUMMARY}

We have reviewed the state of the art in nanostructured dry adhesive fabrication. Innovative fabrication strategies have yielded key insights and as well as increased adhesive performance. However, there is still much to learn about nanostructured dry adhesives, and what is the best hierarchical implementation for strong, durable, self-cleaning adhesives. Although research seems to be headed in the right direction and nanostructured adhesives with adhesion strength greater than the gecko on some surfaces have been reported, the general dry adhesive for both smooth and porous surfaces is still to be developed, and the gecko continues to provide inspiration in this regard. While climbing robots similar in size to the gecko and capable of climbing on both smooth and rough surfaces has often been cited as the target use for nanostructured dry adhesives other industries that may take advantage of reusable residuefree adhesives are temporary signage and security. With these uses in mind, the ability to fabricate large sheets of nanostructured dry adhesives is desired and may be closest to realization using commercially available membranes and sheets for molds.

\section{ACKNOWLEDGMENTS}

This work was financially supported by the Natural Sciences and Engineering Research Council of Canada (NSERC).

\section{REFERENCES}

Autumn, K. (2007). Gecko adhesion: structure, function, and applications. MRS Bull. 32, 473-478. doi:10.1557/mrs2007.80

Autumn, K., Liang, Y. A., Hsieh, S. T., Zesch, W., Chan, W. P., Kenny, T. W., et al. (2000). Adhesive force of a single gecko foot-hair. Nature 405, 681-685. doi:10.1038/35015073

Autumn, K., Majidi, C., Groff, R. E., Dittmore, A., and Fearing, R. (2006a). Effective elastic modulus of isolated gecko setal arrays. J. Exp. Biol. 209, 3558-3568. doi:10.1242/jeb.02469

Autumn, K., Dittmore, A., Santos, D., Spenko, M., and Cutkosky, M. (2006b). Frictional adhesion: a new angle on gecko attachment. J. Exp. Biol. 209(Pt 18), 3569-3579. doi:10.1242/jeb.02486

Autumn, K., Sitti, M., Liang, Y. A., Peattie, A. M., Hansen, W. R., Sponberg, S., et al. (2002). Evidence for van der Waals adhesion in gecko setae. Proc. Natl. Acad. Sci. U.S.A. 99, 12252-12256. doi:10.1073/pnas.192252799

Bhushan, B., and Sayer, R. A. (2007). "Gecko feet: natural attachment systems for smart adhesion," in Applied Scanning Probe Methods VII: Biomimetics and Industrial Applications, eds B. Bhushan, and H. Fuchs (Berlin: Springer), 41-76.

Daltorio, K. A., Gorb, S., Peressadko, A., Horchler, A. D., Ritzmann, R. E., and Quinn, R. D. (2006). "A robot that climbs walls using micro-structured polymer feet," in Climbing and Walking Robots, eds M. O. Tokhi, G. S. Virk, and M. A. Hossain (Berlin: Springer Berlin Heidelberg), 131-138.

Geim, A. K., Dubonos, S. V., Grigorieva, I. V., Novoselov, K. S., Zhukov, A. A., and Shapoval, S. Y. (2003). Microfabricated adhesive mimicking gecko foot-hair. Nat. Mater. 2, 461-463. doi:10.1038/nmat917

Gillies, A. G., and Fearing, R. S. (2011). Shear adhesion strength of thermoplastic gecko-inspired synthetic adhesive exceeds material limits. Langmuir. doi:10.1021/la202085j

Gorb, S., Varenberg, M., Peressadko, A., and Tuma, J. (2007). Biomimetic mushroomshaped fibrillar adhesive microstructure. J R Soc Interface 4, 271-275. doi:10. 1098/rsif.2006.0164

Hansen, W. R., and Autumn, K. (2005). Evidence for self-cleaning in gecko setae. Proc. Natl. Acad. Sci. U.S.A. 102, 385-389. doi:10.1073/pnas.0408304102

Ho, A. Y., Yeo, L. P., Lam, Y. C., and Rodríguez, I. (2011). Fabrication and analysis of gecko-inspired hierarchical polymer nanosetae. ACS Nano 5, 1897-1906. doi:10.1021/nn103191q 
Hu, S., Xia, Z., and Dai, L. (2013). Advanced gecko-foot-mimetic dry adhesives based on carbon nanotubes. Nanoscale 5, 475-486. doi:10.1039/c2nr33027j

Izadi, H., Zhao, B., Han, Y., McManus, N., and Penlidis, A. (2012). Teflon hierarchical nanopillars with dry and wet adhesive properties. J. Polym. Sci. B Polym. Phys. 50, 846-851. doi:10.1002/polb.23076

Izadi, H., Golmakani, M., and Penlidis, A. (2013). Enhanced adhesion and friction by electrostatic interactions of double-level teflon nanopillars. Soft Matter 9, 1985. doi: $10.1039 / \mathrm{c} 2 \mathrm{sm} 27329 \mathrm{~b}$

Jeong, H. E., Lee, J. K., Kim, H. N., Moon, S. H., and Suh, K. Y. (2009). A nontransferring dry adhesive with hierarchical polymer nanohairs. Proc. Natl. Acad. Sci. U.S.A. 106, 5639-5644. doi:10.1073/pnas.0900323106

Jeong, H. E., Lee, J.-K., Kwak, M. K., Moon, S. H., and Suh, K. Y. (2010a). Effect of leaning angle of gecko-inspired slanted polymer nanohairs on dry adhesion. Appl. Phys. Lett. 90, 043704. doi:10.1063/1.3298554

Jeong, H. E., Kwak, M. K., and Suh, K. Y. (2010b). Stretchable, adhesion-tunable dry adhesive by surface wrinkling. Langmuir 26, 2223-2226. doi:10.1021/la904290g

Jeong, H. E., and Suh, K. Y. (2009). Nanohairs and nanotubes: efficient structural elements for gecko-inspired artificial dry adhesives. Nano Today 4, 335-346. doi:10.1016/j.nantod.2009.06.004

Jeong, H. E., and Suh, K.-Y. (2012). Precise tip shape transformation of nanopillars for enhanced dry adhesion strength. Soft Matter 8, 5375-5380. doi:10.1039/ c2sm07440k

Kim, S., Spenko, M., Trujillo, S., Heyneman, B., Santos, D., and Cutkosky, M. R. (2008). Smooth vertical surface climbing with directional adhesion. IEEE Trans. Robot. 24, 65-74. doi:10.1109/TRO.2007.909786

Krahn, J., Liu, Y., Sadeghi, A., and Menon, C. (2011). A tailless Timing Belt Platform (TBCP-II) utilizing dry adhesives with mushroom caps. Smart Mater. Struct. 20, 11. doi:10.1088/0964-1726/20/11/115021

Kustandi, T. S., Samper,V. D., Ng, W. S., Chong, A. S., and Gao, H. (2007). Fabrication of a gecko-like hierarchical fibril array using bonded porous alumina template. J. Micromech. Microeng. 17, N75-N81. doi:10.1088/0960-1317/17/10/N02

Kwak, M. K., Jeong, H.-E., Kim, T., Yoon, H., and Suh, K. Y. (2010). Bio-inspired slanted polymer nanohairs for anisotropic wetting and directional dry adhesives. Soft Matter 6, 1849-1857. doi:10.1039/b924056j

Lee, D. H., Kim, Y., Fearing, R. S., and Maboudian, R. (2011). Effet of fiber geometry on macroscale friction of ordered low-density polyethylene nanofiber arrays. Langmuir 27, 11008-11016. doi:10.1021/la201498u

Lee, D. Y., Lee, D. H., Lee, S. G., and Cho, K. (2012). Hierarchical gecko-inspired nanohairs with a high aspect ratio induced by nanoyielding. Soft Matter 8 , 4905-4910. doi:10.1039/c2sm07319f

Lee, H., and Bushan, B. (2012). Fabrication and characterization of hierarchical nanostructured smart adhesion surfaces. J. Colloid Interface Sci. 372, 231-238. doi:10.1016/j.jcis.2012.01.020

Lee, J., and Fearing, R. S. (2012). Wet self-cleaning of superhydrophobic microfiber adhesives formed from high density polyethylene. Langmuir 28, 15372-15377. doi:10.1021/la303017a

Liu, K., Du, J., Wu, J., and Jiang, L. (2012). Superhydrophobic gecko feet with high adhesive forces towards water and their bio-inspired materials. Nanoscale 4, 768-772. doi:10.1039/clnr11369k

Masuda, H., and Fukuda, K. (1995). Ordered metal nanohole arrays made by a twostep replication of honeycomb structures of anodic alumina. Science 268, 1466. doi:10.1126/science.268.5216.1466
Matschuk, M., and Larsen, N. B. (2013). Injection molding of high aspect ratio sub$100 \mathrm{~nm}$ nanostructure. J. Micromech. Microeng. 23, 025003. doi:10.1088/0960$1317 / 23 / 2 / 025003$

Nielsch, K., Choi, J., Schwirn K., Wehrspohn R. B., and Gosele, U. (2002). Selfordering regimes of porous alumina: the 10 porosity rule. Nano Lett. 2, 677-680. doi:10.1021/nl025537k

Northern, M. T., and Turner, K. L. (2005). A batch fabricated biomimetic dry adhesive. Nanotechnology 16, 1159-1166. doi:10.1088/0957-4484/16/8/030

Palacio, M. L. B., Bhushan, B., and Schricker, S. R. (2013). Gecko-inspired fibril nanostructures for reversible adhesion in biomedical applications. Mater. Lett. 92, 409-412. doi:10.1016/j.matlet.2012.11.023

Rodríguez, I., Lim, C. T., Natarajan, S., Ho, Y. Y., Van, E. L., Elmouelhi, N., et al. (2013). Shear adhesion strength of gecko inspired tapes on surfaces with variable roughness. J. Adhesion 89, 921-936. doi:10.1080/00218464.2013.767198

Röhrig, M., Thiel, M., Worgull, M., and Hölscher, H. (2012). Hierarchical Structures: 3D direct laser writing of nano- and microstructured hierarchical geckomimicking surfaces. Small 8, 3009-3015. doi:10.1002/smll.201200308

Sameoto, D., and Menon, C. (2010). Recent advances in the fabrication and adhesion testing of biomimetic dry adhesives. Smart Mater. Struct. 19, 103001. doi:10.1039/c2nr33027j

Sitti, M., and Fearing, R. S. (2003). Synthetic gecko foot-hair micro/nanostructures as dry adhesives. J. Adhes. Sci. Technol. 17, 1055-1073. doi:10.1163/ 156856103322113788

Song, J., Mengüç, Y., and Sitti, M. (2013). Enhanced fabrication and characterization of gecko-inspired mushroom-tipped microfiber adhesives. J. Adhes. Sci. Technol. 27, 1921-1932. doi:10.1080/01694243.2013.766533

Tsai, Y. C., Wu, M.-D., and Shih, W.-P. (2011). Fabrication and characterization of e-beam photoresist array for biomimetic self-cleaning dry adhesives. Microelectron. Eng. 88, 2126. doi:10.1016/j.mee.2011.01.049

Zhang, J., Shokouhi, B., and Cui, B. (2012). Tilted nanostructure fabrication by electron beam lithography. J. Vac. Sci. Technol. B 30, 06F302. doi:10.1116/1.4754809

Zhou, M., Pesika, N., Zeng, H., Tian, Y., and Israelachvili, J. (2013). Recent advances in gecko adhesion and friction mechanisms and development of gecko-inspired dry adhesive surfaces. Friction 1, 114-129. doi:10.1007/s40544-013-0011-5

Conflict of Interest Statement: The authors declare that the research was conducted in the absence of any commercial or financial relationships that could be construed as a potential conflict of interest.

Received: 10 September 2013; accepted: 04 December 2013; published online: 30 December 2013.

Citation: Pattantyus-Abraham A, Krahn J and Menon C (2013) Recent advances in nanostructured biomimetic dry adhesives. Front. Bioeng. Biotechnol. 1:22. doi: 10.3389/fbioe.2013.00022

This article was submitted to Bionics and Biomimetics, a section of the journal Frontiers in Bioengineering and Biotechnology.

Copyright (c) 2013 Pattantyus-Abraham, Krahn and Menon. This is an open-access article distributed under the terms of the Creative Commons Attribution License (CC $B Y)$. The use, distribution or reproduction in other forums is permitted, provided the original author(s) or licensor are credited and that the original publication in this journal is cited, in accordance with accepted academic practice. No use, distribution or reproduction is permitted which does not comply with these terms. 